\title{
Integrating cryo-fixation and electron microscopy with molecular tools to understand how plants secrete their cell walls
}

\author{
A.L. Samuels, K.H. Rensing, M. Kaneda and D. Bird
}

Department of Botany, University of British Columbia, Vancouver, BC, Canada V6T 1Z4

All plant growth, including agricultural and forestry production, requires cell wall synthesis. Despite its critical importance, there is a major gap in our understanding of cell wall biosynthesis: how are cell wall components, which are made inside the cell, exported to the outside of the cell to build a functional wall? In this presentation, two projects are presented: wax secretion to the plant cuticle and secondary cell wall production during wood formation. Our approach is to integrate cell structure with function using advanced microscopy techniques such as high-pressure freezing/freeze substitution, transmission electron microscopy, cryo-scanning electron microscopy and live cell imaging in combination with molecular biology and biochemical approaches.

The plant cuticle seals the plant surface and is composed of a cutin polyester matrix surrounded by and coated with wax. Wax components are synthesized in the epidermal cells of the plant and secreted to the cell wall. How wax molecules are moved from their sites of synthesis in the endoplasmic reticulum to the cuticle is not known [1]. We identified the first components of the wax

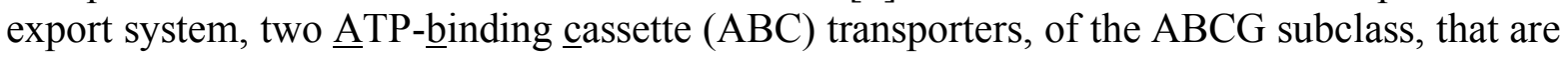
required for wax export from the epidermis to the cuticle of Arabidopsis using mutant studies, cryoSEM and TEM. Mutant cer5 and $w b c 11$ epidermal cells contain rigid inclusions (Figure 1), similar to inclusions found in patients with a human genetic disorder, adrenoleukodystrophy, in which a defect in an ABC transporter leads to lipid build-up in the cytoplasm.

Our studies of the cell structure of developing secondary xylem (wood) have produced unprecedented high-resolution images of wood development by transmission electron microscopy (Figure 2)[2]. The presence of lignin is essential to the vascular integrity of these cells but the mechanism of lignin precursor export is unknown. Using high-pressure freezing/freeze substitution in combination with autoradiography of ${ }^{3} \mathrm{H}$-phenylalanine derivatives, the lignin precursors (monolignols) were localized with light and electron microscopy (Figure 3). This work demonstrates that monolignols were not present in Golgi, suggesting a transporter-mediated mechanism [3].

The goal of this research is to understand the secretion of cell wall components using a cell biology approach to provide the highest quality cell structure information that create the cellular context for underlying molecular, biochemical and physiological processes.

References

[1] A.L. Samuels et al., Annu. Rev. Plant Biol. 59 (2008) 683-707.

[2] C. Courtois-Moreau et al. Plant Journal published on-line Dec. 15, 2008; in press

[3] M. Kaneda et al. Plant Physiol. 147 (2008) 1750-1760.

[4] This research was supported by the Canadian Natural Sciences and Engineering Research Council. This work would not have been possible without the support of the staff of the UBC Bioimaging Facility, especially Derrick Horne for his cryo-SEM assistance. 


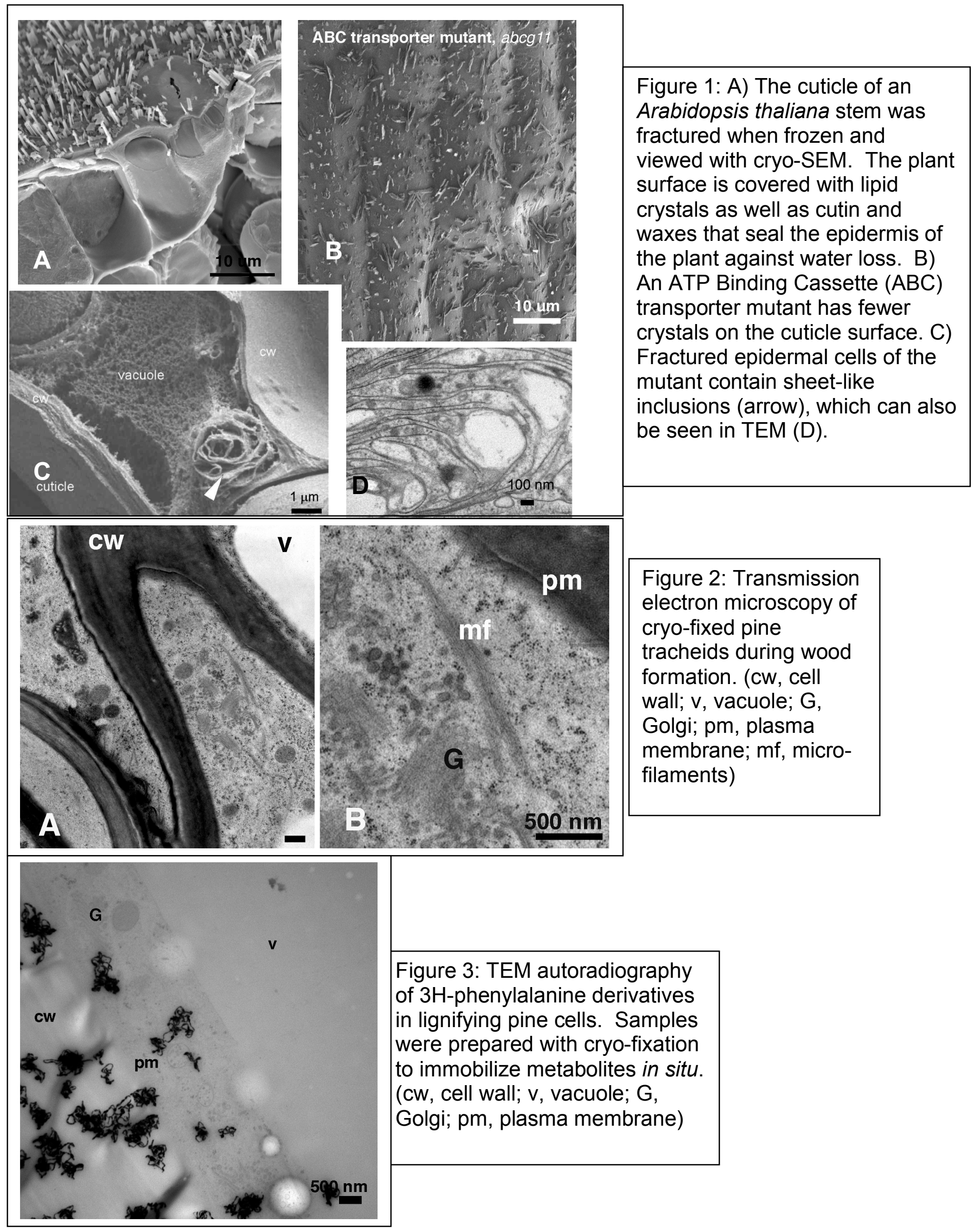

